\title{
Exposición a fluoruros del agua potable en la ciudad de Aguascalientes, México
}

\author{
Rodolfo Trejo-Vázquez ${ }^{1}$ y Adrián Bonilla-Petriciolet ${ }^{1}$
}

RESUMEN Objetivos. Determinar el contenido de fluoruros en todos los pozos de suministro de agua potable de la ciudad de Aguascalientes para establecer el grado de exposición de la población.

Métodos. Se determinó el contenido de fluoruros de los 126 pozos de suministro de agua potable de la ciudad de Aguascalientes, México, con el método SPADNS, conforme a las normativas mexicanas NMX-AA-77-1982 y NMX-014-SSAI-1993. Con los datos obtenidos se crearon mapas de isolíneas de concentración que muestran la distribución de esta variable en los mantos freáticos que abastecen a la ciudad de Aguascalientes y se estimaron las dosis de exposición de los habitantes del área urbana.

Resultados. El error medio de los análisis fue del 3,9\%. Setenta y tres pozos presentaron una concentración $\leq 1,5 \mathrm{mg} / \mathrm{L}$, valor máximo permisible establecido por las normas mexicanas vigentes. Todas las dosis máximas de exposición sobrepasaron el nivel mínimo de riesgo establecido por la ATSDR (Agency for Toxic Substances and Disease Registry). En los menores de un año, incluso la dosis mínima fue ligeramente superior a este nivel de riesgo.

Conclusiones. La estimación de las dosis de exposición a fluoruros ocasionadas por el consumo de agua y su comparación con las obtenidas en otros estados de la República Mexicana indicaron que tal ingesta de fluoruros representa un riesgo potencial para la salud pública de esta área urbana. El contenido de fluoruros del agua para consumo humano en esta ciudad debería reducirse a $0,69 \mathrm{mg} / \mathrm{L}$.

Palabras clave Agua potable, fluoruros, dosis de exposición, México.

Una excesiva bioasimilación de fluoruros en el ser humano puede generar una variedad de padecimientos que incluyen, entre otros, la fluorosis dental y ósea, algunos trastornos gastrointestinales y la disfunción renal (1-4). Varias investigaciones han indicado que estas alteraciones afectan a un

\footnotetext{
Instituto Tecnológico de Aguascalientes, Departamento de Ingeniería Química, Aguascalientes, Ags. México. Toda la correspondencia debe ser enviada a Rodolfo Trejo-Vázquez, Instituto Tecnológico de Aguascalientes. Departamento de Ingeniería Química. Av. López Mateos 1801 Ote. C.P. 20256. Aguascalientes, Ags, México. Correo electrónico: rtrejo@seit.ita.mx
}

considerable porcentaje de la población mundial, siendo la de mayor prevalencia la fluorosis dental. Los fluoruros se encuentran presentes en la mayor parte de los comestibles ingeridos por el ser humano y en algunos dentífricos y medicamentos. Sin embargo, el mayor aporte de fluoruros al organismo procede del agua potable y se ha demostrado que el consumo prolongado de agua con fluoruros disueltos en concentraciones superiores a 1,5 $\mathrm{mg} / \mathrm{L}$ es la principal causa de los trastornos mencionados anteriormente. Esto ha promovido el desarrollo de normas para regular la presencia de estos iones en las que se especifica la concentración máxima permisible que debe contener el agua para consumo humano. En México se ha establecido una concentración máxima de 0,7 $\mathrm{mg} / \mathrm{L}$ para el agua embotellada y de $1,5 \mathrm{mg} / \mathrm{L}$ para el agua distribuida por la red hidráulica $(5,6)$.

En la República Mexicana hay varios estados en los que la gran contaminación del agua potable por fluoruros representa un gran riesgo para la salud pública. Entre ellos se encuentran Durango, San Luis Potosí, Zacatecas, Jalisco, Sonora, Guanajuato y Aguascalientes (7-9). Los resultados de estudios 
anteriores indican que alrededor de 5 millones de personas pertenecientes a estas regiones disponen de acuíferos con un contenido de fluoruros superior al establecido por las normas mexicanas (10). Lamentablemente, el desconocimiento de la distribución y evolución del contenido de fluoruros en el agua extraída del subsuelo limita en gran medida la eficacia de los programas de control de la calidad del agua. En México, tan solo en algunos estados, entre ellos San Luis Potosí y Durango, se han realizado estudios sobre la concentración de fluoruros en las fuentes de suministro de agua potable (11-13). En el Estado de Aguascalientes solo existe un estudio parcial, realizado en 1995, que estableció que las concentraciones presentes en el agua del subsuelo superaban los $2 \mathrm{mg} / \mathrm{L}$ y en algunas áreas alcanzaban los $3 \mathrm{mg} / \mathrm{L}$ (14); sin embargo, los resultados obtenidos no aportan la información necesaria para caracterizar las zonas de alta contaminación de la ciudad.

El objetivo de este trabajo consistió en determinar el contenido de fluoruros en todos los pozos de suministro de agua potable de la ciudad de Aguascalientes para establecer el grado de exposición de la población.

La ciudad de Aguascalientes es el principal municipio y la capital del Estado de Aguascalientes, situado en el centro del país, adyacente a los estados de Jalisco y Zacatecas. Según los datos del último censo, la ciudad tiene aproximadamente 643360 habitantes, de los cuales el $48 \%$ tiene menos de 19 años y el 51\% son mujeres. La ciudad pertenece a la región hidrológica Lerma-Santiago. El acuífero del valle de Aguascalientes es la principal fuente de abastecimiento de agua para consumo humano de esta ciudad y de una porción significativa del Estado de Aguascalientes. El estudio hidrológico más reciente de este acuífero, realizado en 1993 y en el que se efectuó un seguimiento y control de 40 pozos localizados fuera de la ciudad, indica que a esa fecha el agua extraída presentaba una salinidad media con bajas concentraciones de sodio y una cantidad de sólidos disueltos entre $250 \mathrm{y}$ $1050 \mathrm{mg} / \mathrm{L}$ (15). Actualmente la ciu- dad abastece su demanda de casi 500 millones de $\mathrm{m}^{3}$ /año a través de 126 pozos que pertenecen a este acuífero. Hasta el momento, se carece de estudios que caractericen tanto física como químicamente a estas fuentes de abastecimiento de agua. La red hidráulica de la ciudad ha ido creciendo de manera no siempre ordenada desde su inicio, en los años cuarenta del siglo XX, hasta llegar a los 126 pozos que hay en funcionamiento actualmente dentro de la ciudad. El reemplazo de los pozos agotados y el crecimiento de la ciudad han propiciado que, con el tiempo, se hayan creado en la red numerosas interconexiones, muchas de ellas no registradas en los planos. Esto ha ocasionado que no se cuente con información veraz que precise las áreas de influencia de cada pozo ni las conexiones existentes entre ellos.

En este estudio se incluyeron los 126 pozos que abastecen a la ciudad. En cada uno se obtuvieron dos muestras simples, una en julio y otra en agosto de 1999. El segundo muestreo se realizó con objeto de validar los resultados del primero. Los muestreos se realizaron conforme a la norma mexicana (16). Las muestras fueron colectadas en las entradas de las líneas de inyección de cada pozo en la red hidráulica. Se utilizaron envases de polietileno con tapas del mismo material con una capacidad de $250 \mathrm{~mL}$. La temperatura y el $\mathrm{pH}$ de las muestras se determinaron in situ. La cuantificación de fluoruros se realizó en el laboratorio. El intervalo entre la obtención de las muestras y su análisis no superó los 5 días; mientras tanto, fueron mantenidas a una temperatura de $4{ }^{\circ} \mathrm{C}$, sin alcanzar la congelación. El método de análisis utilizado en la cuantificación de fluoruros también fue conforme a lo establecido por la norma mexicana (17), que especifica el uso del método colorimétrico con el reactivo SPADNS (18), reconocido y aceptado por instancias internacionales, siempre que la muestra sea sometida previamente a un proceso de digestión consistente en la destilación en un medio ácido proporcionado por una solución de ácido sulfúrico, con la finalidad de eliminar compuestos que causen interferencia en las cuantificaciones. En este estudio se realizó dicho proceso de digestión.

Los análisis de fluoruros se llevaron a cabo en un espectrofotómetro $\mathrm{Hach}$ $D R / 4000$ con celdas de cuarzo a una longitud de onda de $480 \mathrm{~nm}$. El intervalo de detección de este aparato para una confiabilidad del $95 \%$ fue de 0,02 a 2,0 mg/L. En cada lectura se utilizaron $10 \mathrm{~mL}$ de agua de la muestra y $2 \mathrm{~mL}$ del reactivo SPADNS. La precisión del equipo fue de 0,01 mg/L. La cuantificación de fluoruros en cada muestra se realizó por duplicado, anotándose la media de ambas lecturas, siempre que presentaran una discrepancia $<5 \%$. Cuando la discrepancia fue superior al 5\%, se repitieron las mediciones tomándose nuevas alícuotas de la muestra.

Después de calibrar el aparato se analizaron varias muestras (estándares) con concentraciones conocidas entre $0,5 \mathrm{mg} / \mathrm{L}$ y $15 \mathrm{mg} / \mathrm{L}$ para verificar su exactitud y revisar los efectos tanto de la curva de calibración interna del aparato como de las diluciones, cuando fueron necesarias. El error medio obtenido fue del 3,9\%, ajustando los datos de la curva de calibración a la siguiente expresión:

$$
\begin{aligned}
{\left[F^{-}\right]_{S T D} } & =\beta_{0}+\beta_{1}\left[F^{-}\right]_{\text {Hach }} \\
& +\beta_{2}\left[F^{-}\right]_{\text {Hach }}^{2}
\end{aligned}
$$

donde $\left[F^{-}\right]_{S T D}$ representa la concentración de fluoruros del estándar; $\left[\mathrm{F}^{-}\right]_{\text {Hach }}$ la concentración registrada por el aparato, y $\beta_{0}, \beta_{1}$ y $\beta_{2}$ los coeficientes de ajuste. El coeficiente de determinación $\left(R^{2}\right)$ del modelo fue de 0,998. El error se definió como (19):

Error $=\frac{\left[F^{-}\right]_{S T D}-\left[F^{-}\right]_{E S T}}{\left[F^{-}\right]_{S T D}} * 100$

donde $\left[F^{-}\right]_{\text {est }}$ es la concentración de fluoruros estimada por la expresión de segundo orden utilizada en el ajuste de los datos de la curva de calibración. Respecto al proceso de digestión, la re- 
cuperación de fluoruros de las muestras fue superior al $97 \%$.

Para generar los diagramas de distribución de la concentración de fluoruros en las fuentes de abastecimiento de agua de la ciudad se utilizaron los resultados de los análisis químicos en los dos muestreos. Como no hay estudios sobre las características fisicoquímicas de los 126 pozos de esta ciudad, en la generación de estos diagramas solo se consideraron las coordenadas geográficas de los puntos de muestreo. Los diagramas se desarrollaron con el programa informático Surfer ${ }^{\odot}$ (20).

Para calcular las dosis teóricas de exposición de la población local se utilizaron las concentraciones mínima y máxima encontradas en este estudio, aplicando la fórmula:

$$
D E=\frac{\left[F^{-}\right] C_{H_{2} \mathrm{O}}}{M_{\text {ind }}}
$$

donde $D E$ es la dosis de exposición a fluoruros, en $\mathrm{mg} / \mathrm{kg} /$ día; $\left[\mathrm{F}^{-}\right]$la concentración de este ion en el agua potable, en mg/L; $C_{H 2 O}$ el consumo de agua, en $L /$ día, y $M_{\text {ind }}$ la masa corporal del individuo, en $\mathrm{kg}$. Siguiendo criterios establecidos en la literatura (12), en estas estimaciones se emplearon los valores estándar utilizados por el Instituto Mexicano del Seguro Social (I. Landín Miranda, Comunicación personal, 2000) para la masa corporal y la ingestión de agua de los individuos y se consideró que la biodisponibilidad de fluoruros es total y que el agua potable fue la única fuente de ingestión de fluoruros.

La referencia para comparar estas dosis teóricas fue el nivel mínimo de riesgo calculado por la Agency for Toxic Substances and Disease Registry (ATSDR) (21), que es de $0,05 \mathrm{mg} / \mathrm{kg} /$ día para una exposición oral crónica. Este valor ha sido considerado como el umbral tóxico que puede ocasionar la aparición de fluorosis dental y fracturas óseas en los individuos expuestos.

Las concentraciones medias globales de los dos muestreos se resumen en la figura 1. La variación media entre las
FIGURA 1. Distribución de frecuencias de la concentración de fluoruros en los pozos que abastecen de agua a la ciudad de Aguascalientes. Aguascalientes, México, 1999

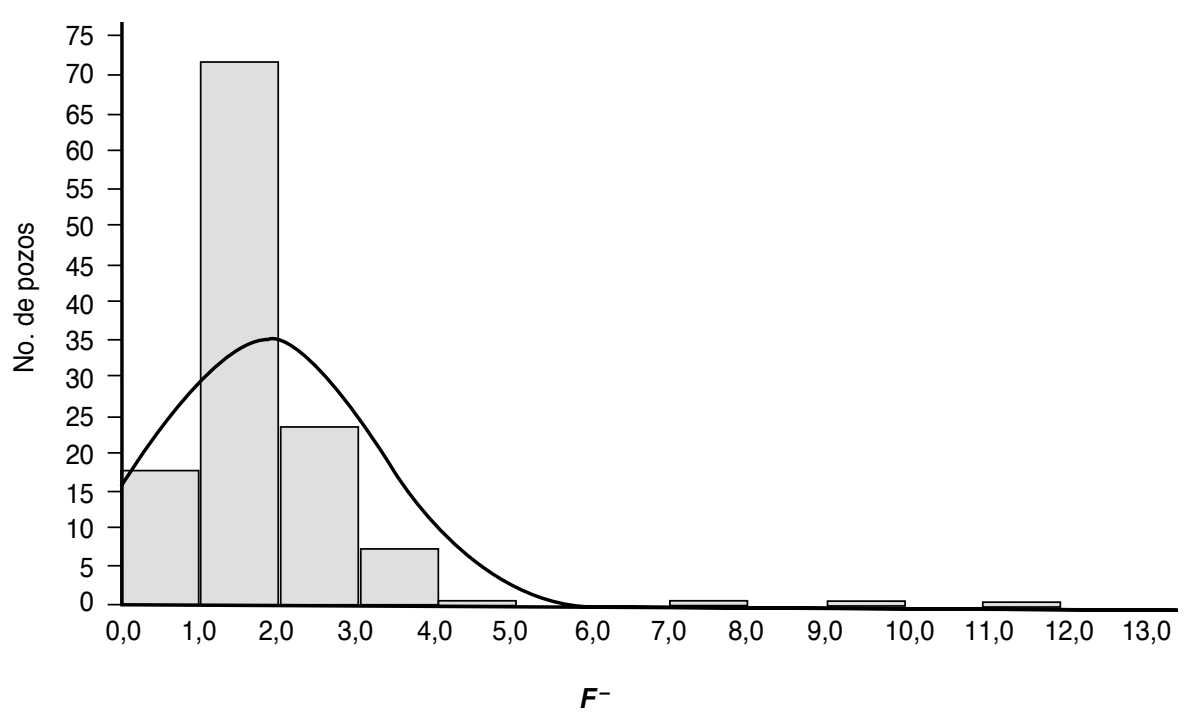

concentraciones determinadas en los dos muestreos fue del $7,05 \%$, sin que existiera una tendencia definida, es decir, en algunos pozos la concentración aumentó y en otros disminuyó. Las concentraciones medias mínima y máxima encontradas en los pozos analizados fueron de $0,53 \mathrm{mg} / \mathrm{L}$ y 11,31 $\mathrm{mg} / \mathrm{L}$, respectivamente. La concentración media total fue de $1,82 \mathrm{mg} / \mathrm{L}$ con una desviación estándar de 1,42 mg/L. En todos los pozos el $\mathrm{pH}$ fue cercano a 7. De los 126 pozos analizados, 73 $(57,9 \%)$ presentaron concentraciones $\leq 1,5 \mathrm{mg} / \mathrm{L}$, valor máximo permitido por la norma mexicana NOM-127SSA1-1994. Tres pozos presentaron una grave contaminación por fluoruros, con concentraciones que superaron los $4,5 \mathrm{mg} / \mathrm{L}$. Los 50 pozos restantes presentaron concentraciones que oscilaron entre 1,51 y $4,5 \mathrm{mg} / \mathrm{L}$.

Como no existió una diferencia significativa entre los contenidos de fluoruros obtenidos en los dos muestreos, los mapas de distribución de la concentración de fluoruros se generaron con los valores medios globales. Como se puede observar en la figura 2 , en la mayor parte de la ciudad, el acuífero del valle de Aguascalientes presenta una concentración de fluoruros $\leq 1,5$ $\mathrm{mg} / \mathrm{L}$. Es en la región sureste de la ciudad donde se presentan las mayores concentraciones de fluoruros. Lamentablemente, la carencia de información sobre las características de los pozos, como la estratificación del subsuelo, entre otras variables, no permite identificar las posibles causas. Esta situación no explica la alta prevalencia de fluorosis dental grave en la población, independientemente de que viva cerca o lejos de los pozos con alta concentración de fluoruros, detectada en estudios de campo por el Instituto de Salud del Estado de Aguascalientes (C. Márquez Algara, Comunicación personal, 2000). Para explicar esto se pensó en un posible efecto de mezclado hidráulico por interconexiones entre pozos. En efecto, los caudales de los pozos con altos contenidos de fluoruros son considerables, y a menudo se hacen interconexiones con ellos para abastecer la demanda de diversas zonas de la ciudad. Para comprobar esta suposición se seleccionaron de forma aleatoria 10 pozos y se determinó la concentración de fluoruros a la entrada de domicilios cercanos a ellos, encontrándose discrepancias de magnitudes diversas, que llegaron al doble o más de la concentración de fluoruros esperada conforme al pozo.

La propuesta más lógica, cerrar los tres pozos con mayores concentraciones de fluoruros, es impracticable, ya 
FIGURA 2. Distribución geográfica de la concentración de fluoruros en el agua potable de la ciudad de Aguascalientes. Aguascalientes, México, 1999

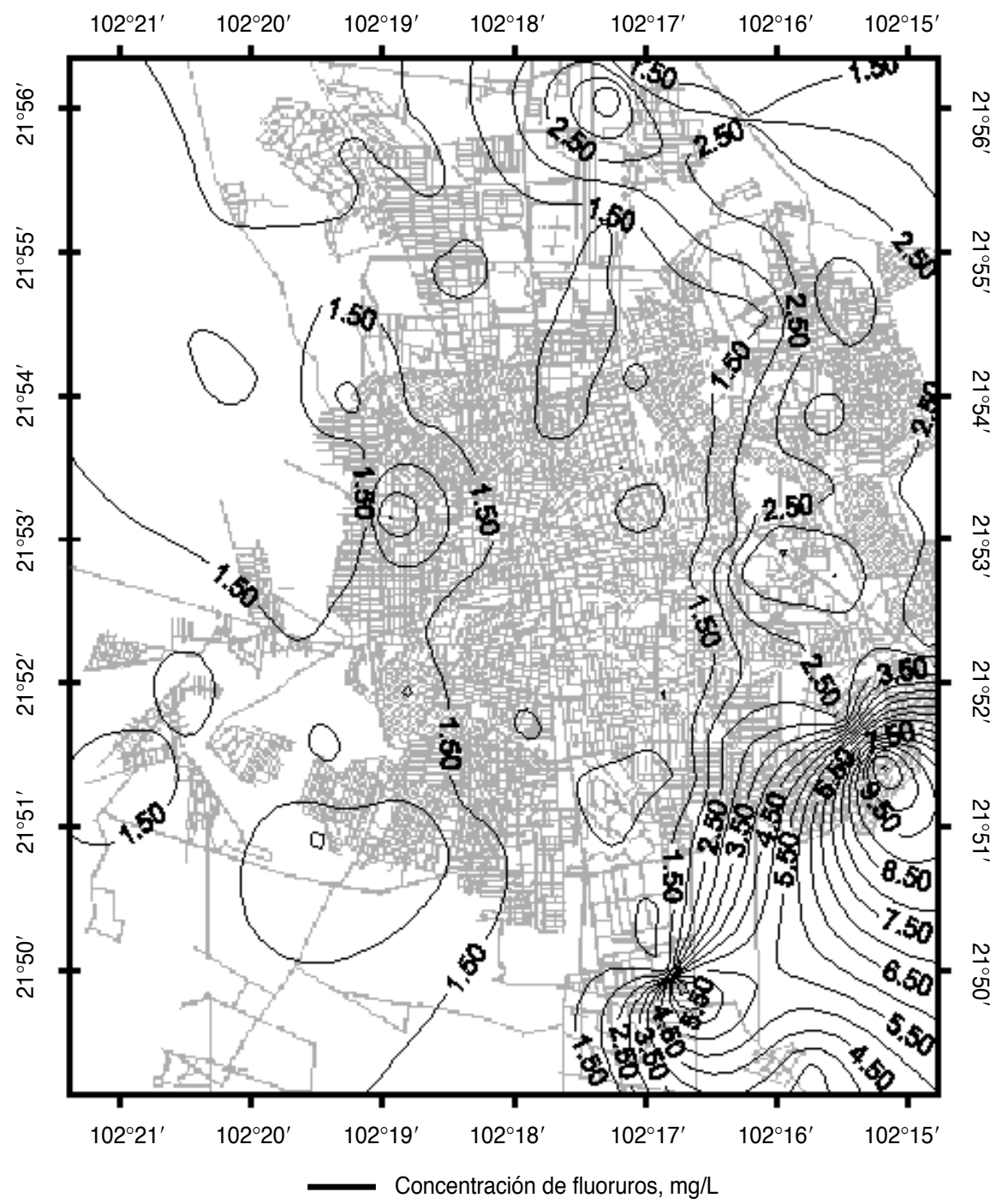

que sin su aportación no se podrían suplir las necesidades de agua de la ciudad y no existen posibilidades de abrir nuevos pozos en otros sitios, pues las autoridades han prohibido la perforación de nuevos pozos en el área urbana a causa de los agrietamientos y hundimientos del terreno que se han presentado en los últimos años. No queda sino recomendar que el agua extraída de estos tres pozos sea tratada de alguna manera, a fin de reducir su contenido de fluoruros.

El cuadro 1 muestra las estimaciones de la ingesta de fluoruros de la población local expuesta. Se puede obser- var que todas las dosis máximas de exposición sobrepasan el nivel mínimo de riesgo de la ATSDR. Por otra parte, para el caso de los niños menores de un año, la ingesta mínima de fluoruros es ligeramente superior a este nivel de riesgo.

Con objeto de ponderar los posibles daños para la salud pública de la población expuesta, se realizó una comparación de las ingestas teóricas obtenidas en este estudio con las descritas para el estado vecino de San Luis Potosí (cuadro 2), en el que se ha llevado a cabo una serie de estudios epidemiológicos que han establecido una signi- ficativa prevalencia de fluorosis dental en sus residentes $(10,12)$. Por ejemplo, en esta ciudad se ha determinado que en las zonas con dosis de exposición $>0,05 \mathrm{mg} / \mathrm{kg} /$ día la prevalencia de fluorosis dental moderada a grave es del $84 \%$ (12). Si se considera que ambas ciudades presentan grandes similitudes y que en Aguascalientes hay mayores dosis de exposición, se justifica el hecho de que esta ciudad presente también una alta prevalencia de fluorosis dental en su población. Sin embargo, no existen aún suficientes estudios epidemiológicos que permitan establecer el estado clínico de la población expuesta, tanto de forma global como por estratos.

Aunque el consumo de agua potable es considerado como la fuente más importante de ingestión de fluoruros, no es la única, ya que existen otras derivadas del consumo de productos dentífricos, medicamentos, bebidas embotelladas y comestibles. Sin embargo, no hay en esta región estudios que determinen la contribución de cada una de estas fuentes a la ingesta total de fluoruros, con objeto de corregir las dosis de exposición calculadas. Además, otro factor que debe ser considerado en la estimación de las dosis teóricas es el consumo de agua hervida. En México, un importante porcentaje de la población consume agua hervida y esto puede incrementar significativamente la concentración de fluoruros, debido a la disminución de volumen. Teniendo en cuenta todos estos factores, se infiere que las exposiciones reales son superiores a los valores estimados en este trabajo.

Partiendo de la proporción de niños y adultos existente en la ciudad, el peso medio de ambos grupos de individuos y su consumo medio de agua, se estimó el contenido máximo permisible de fluoruros del agua extraída de los pozos que abastecen a la ciudad para evitar problemas de salud pública asociados con la ingestión crónica de fluoruros. Considerando que las dosis de exposición son mayores para los individuos de menor edad, en estos cálculos solo se incluyeron los menores de 12 años, agrupados como en los cuadros 1 y 2 . Nuevamente se 
CUADRO 1. Ingesta estimada de fluoruros derivada únicamente del consumo de agua de la red en la ciudad de Aguascalientes. Aguascalientes, México, 1999

\begin{tabular}{|c|c|c|c|c|}
\hline \multirow[b]{2}{*}{$\begin{array}{l}\text { Edad } \\
\text { (años) }\end{array}$} & \multicolumn{2}{|c|}{ Características del individuo } & \multicolumn{2}{|c|}{$\begin{array}{c}\text { Dosis de exposición } \\
\text { (mg/kg/día) }\end{array}$} \\
\hline & $\begin{array}{l}\text { Peso }^{a} \\
(\mathrm{~kg})\end{array}$ & $\begin{array}{c}\text { Consumo de agua }{ }^{a} \\
\text { (L) }\end{array}$ & Mínima & Máxima \\
\hline$<1$ & 6,98 & 1 & 0,0759 & 1,6196 \\
\hline 1 a 2 & 12,31 & 1 & 0,0431 & 0,9188 \\
\hline 3 a 4 & 16,59 & 1 & 0,0320 & 0,6818 \\
\hline 5 a 6 & 20,53 & 1 & 0,0258 & 0,5508 \\
\hline 7 a 8 & 26,01 & 1 & 0,0204 & 0,4349 \\
\hline 9 a 10 & 36,64 & 1 & 0,0145 & 0,3087 \\
\hline 11 a 12 & 42,51 & 2 & 0,0249 & 0,5322 \\
\hline 13 a 14 & 52,36 & 2 & 0,0202 & 0,4321 \\
\hline 15 a 17 & 59,10 & 2 & 0,0179 & 0,3827 \\
\hline$>18$ & 70,0 & 2 & 0,0151 & 0,3231 \\
\hline
\end{tabular}

a Valores medios utilizados por el Instituto Mexicano del Seguro Social (I. Landín Miranda, Comunicación personal, 2000).

CUADRO 2. Ingesta estimada de fluoruros por el consumo de agua potable en la ciudad de San Luis Potosí

\begin{tabular}{lcc}
\hline $\begin{array}{c}\text { Edad } \\
\text { (años) }\end{array}$ & $\begin{array}{c}\text { Ingesta mínima } \\
\text { (mg/kg/día) }\end{array}$ & $\begin{array}{c}\text { Ingesta máxima } \\
\text { (mg/kg/día) }\end{array}$ \\
\hline <1 & 0,1432 & 0,8019 \\
1 a 2 & 0,0812 & 0,4549 \\
3 a 4 & 0,0603 & 0,3376 \\
5 a 6 & 0,0487 & 0,2727 \\
7 a 8 & 0,0385 & 0,2153 \\
9 a 10 & 0,0273 & 0,1528 \\
11 a 12 & 0,0471 & 0,2635 \\
13 a 14 & 0,0382 & 0,2139 \\
15 a 17 & 0,0338 & 0,1895 \\
> 18 & 0,0286 & 0,1600 \\
\hline
\end{tabular}

Fuente: referencias 10 y 12.

consideró que el agua era la única fuente de fluoruros. La estimación se realizó con la siguiente fórmula:

$$
\left[F^{-}\right]_{\text {recomendado }}=\frac{N M R_{A T S D R}}{\sum_{i} \frac{f_{i} C_{i}}{m_{i}}}
$$

donde $\left[\mathrm{F}^{-}\right]_{\text {recomendado }}$ es la concentración de fluoruros recomendada para esta ciudad; $N M R_{A T S D R}$ el nivel mínimo de riesgo de la ATSDR; $f_{i}$ la frecuencia de población del i-ésimo grupo de edad de los individuos considerados en el cálculo, y $m_{i}$ y $C_{\mathrm{i}}$ la masa corporal y el consumo medio de agua de los grupos de individuos. De esta manera se determinó que el contenido de fluoruros del agua potable que reciben en sus casas los habitantes de la ciudad debe ser $<0,86 \mathrm{mg} / \mathrm{L}$. En caso de que el agua consumida se hirviera, y estimando una pérdida de volumen del $20 \%$, la concentración debería ser $<0,69 \mathrm{mg} / \mathrm{L}$. Finalmente, el cuadro 3 muestra las concentraciones puntuales mínima y máxima registradas por un investigador mexicano en varias ciudades de la República Mexicana (22), que demuestran que gran parte del territorio mexicano dispone de fuentes de suministro de agua potable con un alto contenido de fluoruros. De lo anterior se infiere la presencia de un problema de salud pública nacional, del cual es partícipe la ciudad de Aguascalientes.

En resumen, el contenido de fluoruros del $58 \%$ de los 126 pozos de abas-

CUADRO 3. Contenido de fluoruros mínimo y máximo presente en el agua potable de algunas ciudades del territorio mexicano

\begin{tabular}{lcc}
\hline \multicolumn{1}{c}{ Ciudad } & $\begin{array}{c}\text { Concentración } \\
\text { mínima } \\
\text { (mg/L) }\end{array}$ & $\begin{array}{c}\text { Concentración } \\
\text { máxima } \\
(\mathrm{mg} / \mathrm{L})\end{array}$ \\
\hline Hermosillo & 0,2 & 7,8 \\
San Luis Potosí & 0,3 & 5,8 \\
Salamanca & 0,6 & 6,5 \\
Durango & 1,0 & 5,6 \\
\hline
\end{tabular}

Fuente: referencia 22. tecimiento de agua potable de la ciudad de Aguascalientes se encuentra dentro de los límites establecidos por la norma mexicana. Solo tres pozos presentaron una seria contaminación por fluoruros, que superaba los valores permitidos en más de un $200 \%$. Sin embargo, una gran parte de la ciudad dispone de agua con altos contenidos de fluoruros debido a interconexiones hidráulicas realizadas con el fin de abastecer a toda la población. La dosis de exposición estimada y su comparación con los resultados descritos en una región endémica de México confirman la presencia de un problema de salud pública en esta área urbana. Dadas las características de la población de Aguascalientes, creemos que es necesario reducir a $0,69 \mathrm{mg} / \mathrm{L}$ el contenido de fluoruros del agua para consumo humano en esta ciudad.

Para complementar el presente estudio se recomienda realizar estudios epidemiológicos con objeto de establecer el tipo y prevalencia de las lesiones originadas por dicha ingesta, así como su relación con determinadas áreas geográficas de la ciudad. Además, al menos tres pozos requieren la aplicación de dispositivos para reducir el contenido de fluoruros del agua. Asimismo, hacen falta programas de evaluación y control del contenido de fluoruros de las fuentes de agua para consumo humano de esta ciudad. Hace falta también llevar a cabo una actualización del estado de la red hidráulica actual con todas sus interconexiones, cancelaciones y modificaciones, incluidos los flujos que se conectan y sus procedencias, así como un estudio hidrológico e hidrobiológico, además de un estudio estratigráfico para actualizar y complementar la información existente acerca de las fuentes de suministro de agua potable de esta ciudad.

Agradecimientos. Los autores agradecen el apoyo económico del Consejo del Sistema Nacional de Educación Tecnológica (COSNET) a través del Convenio 681.99-P y de la empresa Concesionaria de Aguas de Aguascalientes S.A. de C.V. 
1. Chinoy NJ. Effects of fluoride on physiology of some animals and human beings. Indian J Environ Toxicol 1991;1:17-32.

2. Hillier S, Cooper C, Kellingray S, Russell G, Hughes $\mathrm{H}$, Coggon D. Fluoride in drinking water and risk of hip fracture in the UK: a case control study. Lancet 2000;355(9200):265-269.

3. Mascarenhas AK, Burt BA. Fluorosis risk from early exposure to fluoride toothpaste. Fluoride 1999;32:35.

4. Takahashi K. Fluoride-linked Down syndrome births and their estimated ocurrence due to water fluoridation. Fluoride 1998:31: 61-73.

5. Norma Oficial Mexicana NOM-041-SSA11993, Bienes y Servicios. Agua purificada y envasada. Especificaciones sanitarias. México, DF; Diario Oficial de la Federación, 24 de marzo de 1995.

6. Norma Oficial Mexicana NOM-127-SSA11994. Salud ambiental, agua para uso y consumo humano. Límites permisibles de calidad $\mathrm{y}$ tratamientos a que debe someterse el agua para su potabilización. México, DF: Diario Oficial de la Federación, 18 de enero de 1996.

7. Cervantes González ME, Ortiz Burgos J. Concentración de flúor en ppm de los pozos de agua potable en la Cd. de Salamanca, Gto. Revista Médica de Aguascalientes 1997;4:15A.

8. Grijalvo Haro MI, Valenzuela Quintanar AI, Silveira Gramont MI, Benítez Salas MA. Concentración de fluoruros en agua de consumo y su relación con la salud dental en el estado de Sonora, México. Sonora, México: Centro de Investigación en Alimentos y Desarrollo A.C.; 1993.

9. Medellín Milán P, Alfaro de la Torre MC, Sarabia Meléndez I, De Lira Santillan A, Nieto
Ahumada B. Fluoruros en aguas de consumo en la ciudad de San Luis Potosí y zona conurbana con el municipio de Soledad. Oaxaca, México: Congreso Nacional de Ingeniería Sanitaria y Ambiental A.C.; 1990.

10. Díaz-Barriga F, Navarro Quezada A, Grijalva MI, Grimaldo M, Loyola Rodriguez JP, Deogracias Ortiz M. Endemic fluorosis in México. Fluoride 1997;30:233-239.

11. Trejo R, Alarcón MT, Martínez Y, Romero P, Salvador J. Niveles de fluoruros en el agua de los pozos de la ciudad de Durango. Ingeniería Hidráulica en México 1997;12:51-57.

12. Ortiz D, Castro L, Turrubiates F, Milán J, Díaz-Barriga F. Assesment of the exposure to fluoride from drinking water in Durango, México, using a Geographic Information System. Fluoride 1998:31:183-187.

13. Díaz-Barriga F, Leyva R, Quistián J, LoyolaRodríguez JP, Pozos A, Grimaldo M. Endemic fluorosis in San Luis Potosí, México. IV Sources of fluoride exposure. Fluoride 1997; 30:219-222.

14. Flúor en la Ciudad de Aguascalientes, 2da Etapa. Aguascalientes, México: Comisión Nacional del Agua, Gerencia Estatal en Aguascalientes; 1995.

15. México, Instituto Nacional de Estadística, Geografía e Informática. Estudio Hidrológico del Estado de Aguascalientes. Aguascalientes, México: INEGI; 1993

16. Norma Oficial Mexicana NOM 014-SSA11993. Procedimientos para el muestreo de agua para uso y consumo humano en sistemas de abastecimiento de agua públicos y privados. México, DF: Diario Oficial de la Federación, 8 de diciembre de 1994 .
17. Norma Oficial Mexicana NMX-AA-077-1982. Análisis de Agua. Determinación de fluoruros. Secretaría de Comercio y Fomento Industrial. México, DF: General de Normas, 6 de enero de 1982

18. American Public Health Association. Standard Methods for the Examination of Water and Wastewater. Clesceri IS, Greenberg AE, Eaton ADS (Eds.), 19a. ed. Washington, DC: APHA; 1995

19. Lipka J. Computaciones gráficas y mecánicas México DF: Compañía Editorial Continental S.A.; 1996.

20. Surfer for Windows Versión 6.0 [Programa de computadora], User's Guide. Golden, CO Golden Software, Inc; 1997.

21. U.S., Agency for Toxic Substances and Disease Registry. Department of Health and Human Services, Public Health Service. Toxicological profile for fluoride, hydrogen fluoride, and fluorine (F). Altlanta, USA: ATSDR; 1993.

22. Díaz-Barriga F. Análisis de la contaminación por compuestos tóxicos en el acuífero que abastece a la ciudad de San Luis Potosí. Querétaro, México: Sistema de Investigación $\mathrm{Mi}$ guel Hidalgo, Cuaderno de Trabajo, Consejo Nacional de Ciencia y Tecnología; 2000

Manuscrito recibido el 23 de febrero de 2001 y aceptado para publicación, tras revisión, el 31 de mayo de 2001

ABSTRACT Objective. Determine the fluoride content in all the wells that supply drinking water to the city of Aguascalientes, Mexico, in order to establish the population's degree of exposure.

\section{Exposure to fluorides from drinking water in the city of Aguascalientes, Mexico}

Methods. The fluoride content of the 126 wells that supply drinking water to the city of Aguascalientes was determined, using the SPADNS method, in accordance with two Mexican regulations, NMX-AA-77-1982 and NMX-014-SSAI-1993. Using that data, we created fluoride isopleth maps showing the distribution of fluoride concentrations in the water supplies for the city of Aguascalientes. We also estimated exposure doses for the city's inhabitants.

Results. The mean analysis uncertainty was 3.9\%. Seventy-three wells had a fluoride concentration of $\leq 1.5 \mathrm{mg} / \mathrm{L}$, which was the maximum permissible value set by the Mexican standards then in effect. All the maximum exposure doses surpassed the minimum risk level set by Agency for Toxic Substances and Disease Registry (ATSDR) of the Department of Health and Human Services of the United States of America. In the children under 1 year of age, even the minimum dose was slightly higher than the ATSDR risk level.

Conclusions. From estimating the fluoride exposure doses caused by water consumption in the city of Aguascalientes and comparing those doses with ones from other states in Mexico, we concluded that the fluoride intake in Aguascalientes represents a potential risk for inhabitants' health. The fluoride content of the city's drinking water should be reduced to $0.69 \mathrm{mg} / \mathrm{L}$. 